\title{
An IoT Framework for Addressing Parents Concerns about Safety of School Going Children
}

\author{
Poonam Gupta $^{1}$, D D Shah ${ }^{2}$, K V V Satyanarayana ${ }^{3}$ \\ ${ }^{1,3}$ Dept. of CSE, K L University, Koneru Laksamiah Education Foundation, Vijaywada, India \\ ${ }^{2}$ G. H. Raisoni College of Engineering and Management, Pune, India
}

\begin{tabular}{l}
\hline \hline Article Info \\
\hline Article history: \\
Received Mar 10, 2016 \\
Revised Sep 7, 2016 \\
Accepted Sep 21, 2016
\end{tabular}

Keyword:

Internet of things

Safety monitoring

Soft computing

Ubiquitous computing

\begin{abstract}
In this paper, we have proposed a novel application using Internet of things (IOT). This application is focused to address the concerns of the parents towards their school going kids. Mainly the concerns of the parents are to ensure the safety of their kids in school bus as well as at school premises. In this paper, we have tried to provide detailed technical implementation about how different sensing, communication technologies clubbing together provides a platform in terms of IoT, where proposed application can be implemented to ensure safety of school going children as it is the priority and concern for parents. In Proposed application, parents get notification when his child boards the bus for school and gets down the bus at home's doorstep. Parents also get notification when child enters his Class Room first time in a day. Parents any time can access the location his child or school bus in which his child is travelling. In case of emergency, child can disseminate the signal to parents / Single point of contact (SPOC) at school to make them aware about emergency.
\end{abstract}

Copyright $(0) 2016$ Institute of Advanced Engineering and Science. All rights reserved.

\section{Corresponding Author:}

Poonam Gupta,

Departement of Computer Engineering \& Science,

K. L. University,

Vijaywada, India.

Email: poonam77gupta@gmail.com

\section{INTRODUCTION}

Internet of Things is a novel paradigm where the objects, animals, people etc. can be uniquely identified and can communicate with each other over the Internet without any human intervention or human to human or human to computer interaction. The "Internet of Things" term was firstly coined by Kevin Ashton as a title of his presentation in 1999 [1]. The basic concept of the Internet of Things paradigm is that the objects can be sensors, actuators, mobile phones or Radio Frequency Identification (RFID) Tags etc. which can be uniquely identified with unique addressing schemes and are able to interact with each other and co-operate with their neighbours to reach common goals [2].There aren't any standard definitions for the Internet of Things paradigm, as it is a very novel concept. The following definitions are have defined by different researchers:

Definition by [3]: "Things have identities and virtual personalities operating in smart spaces using intelligent interfaces to connect and communicate within social, environment, and user contexts."

Definition by [4]: "The Internet of Things allows people and things to be connected Anytime, Anyplace, with Anything and Anyone, ideally using Any path/network and Any service.”

The Internet of Things paradigm can be viewed as one paradigm, many visions [2]. The visions can be classified as Internet-oriented, Things-oriented and Semantic-oriented. Internet-oriented Visions mainly focuses on trying to uniquely identify the things and focuses on IP for Smart Objects and Web of Things. The Things-oriented vision mainly focuses on the objects such as sensors, actuators etc. While the 
Semantic-oriented vision mainly focuses on the Smart Internet of Things which are capable of taking independent decisions by reasoning over data and machine learning.

With the Internet of Things paradigm there can be potentially huge number of applications in multiple domains such as Transportation and Logistics, Health Care, Smart Home and Office etc. One of the applications is smart and sustainable city. Many factors and challenges like job creation, environmental sustainability, economic growth and better public services etc. are considered in making the city smart with the help of Internet of Things. "Digital Urbanism [5]" is a term which is currently being adopted by the city planners and architects, engineers etc. to realise the smart and sustainable cities.

Another interesting application of the Internet of Things is "The Talking Museum [6]". This project makes the objects of a museum exhibition able to "talk" during the user's visit. The objects really tell a story using multimedia facilities. Virtualisation of floricultural supply chains [7] creates a virtual supply chain management system in the floricultural sector.

We apply IoT for monitoring safety of the school students. The parents can monitor their child with the help of a mobile app. Previously there has been research done on this topic [8] which focused more on the types of networks and standards which could be used to realise it. Many attempts have been made previously [9-10] where the school has tried to track the locations of the students, which have been opposed by many privacy advocacy groups. So in this project we only given the parents of the student all rights to access the location while very limited rights to the school. The School can only get the access of the student's location in case of an emergency.

\section{PROPOSED DETAILS}

Safety of school going students is the priority to parents and responsibility of school management and authority. Right from boarding of school bus, reaching to school premise then entering to his class room, studying, playing at school, then again boarding a bus and returning back to home and ultimately getting down from bus should be done safely and in secure environment, and safety and security is to be ensured at every point of time. Parents are always concerned about knowing the status of his ward at any point of time we have proposed a novelty application which ensures safety of school going students.

To implement the proposed application and provide IoT, we thought of clubbing the available infrastructure such as mobiles, servers, CCTV camera and other necessary infrastructure like Gateway Server.We need to understand the expectations and perspective of different users: Parents, Staff, Student, SPOC and Vehicle. Figure 1 shows the use case diagram.

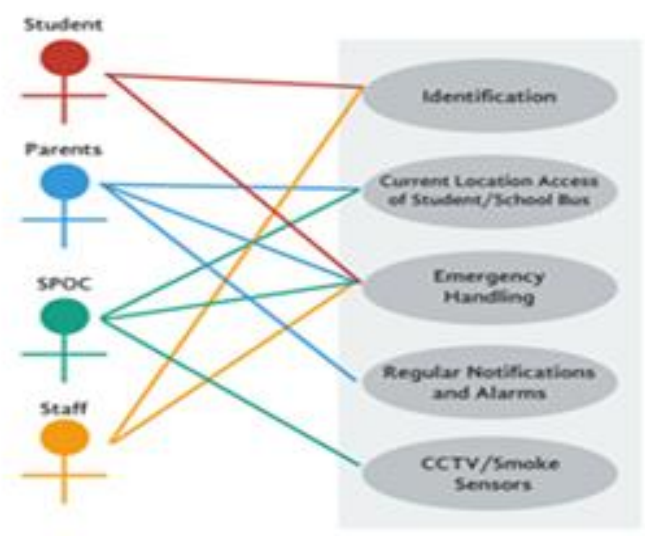

Figure 1. Use Case Diagram

Parents:

a. When student boards a school bus (say at time t1), enters the class room first time for a day (say at time t2) registered mobile should get notifications $n 1$ and $n 2$ respectively.

b. If parent gets $\mathrm{n} 1$ notification at $\mathrm{t} 1$ then for every student some time say is defined as $\mathrm{t}$, waiting time is fixed in system. Suppose if mobile app does not receive $\mathrm{n} 2$ after $\mathrm{t}$ then without intervention of parent, mobile app itself should check the current location of school bus if the school bus is on the route towards school then parent should get this information through notification. In case school bus has reached to school then current location of the student must be checked. 
c. If parents want to access the location of his ward he should be able to get it and should able to find whether student is in Classroom / Ground / AVR / Lab.

Student Staff / AS an Actor:

a. Unique identification location access

b. In case of emergency, student must be able to inform and connect to parents /SPOC at school. Parents and SPOC should get an alarm as notification for this situation.

Vehicle as an Actor:

a. Able to get count the students seating in bus.

b. Unauthorized person should not be allowed to enter the bus.

c. Reply to parents query in care bus is late.

d. Pick up and Drop Route and time is fixed. In case of delay notification needs to be sent to parents as well as schools SPOC.

School:

a. 2-Tier security. All Authorized vehicle identification will be done at Gate. All CR, LAB,AV Rooms, Faculty Rooms will have unit to read the tags.

b. CCTV Cameras are installed at different locations to monitor all activities with SPOC having its control.

c. Smoke detectors are also installed to detect the fire incidents at school. It also gives alarm in care of fire detection and sends notification to SPOC.

d. In case of emergency alert, the SPOC should be able access the location of students.

\section{SYSTEM MODEL}

Various sensors such as RFID Readers, CCTV Cameras, and Smoke Detectors will be deployed on the School Bus and in different locations at School. The students and staff will have a RFID tag attached on their ID card which will uniquely identify the students and staff as well as can provide locations with GPS built-in the RFID tags. At Level 1 of the proposed system is sensing and acquiring the Data. All the sensing devices provide certain type of data. This data is of no use unless it is processed to get the information.

At Level 2 different sink nodes i.e. Gateway Devices are developed and installed at School Bus and in different locations at School.These sink nodes collect all the data and model it in such a way it can cater the requirement of Application i.e. it can provide appropriate services to applications. This information is sent to the main Server, which also does some kind of processing to this input information. This is the Level 2 of the proposed system.

At Level 3, we have apps installed on the mobile devices of parents, staff and SPOC. The mobile apps installed on the Parent's mobile phones will get the notifications once their ward boards the School Bus, enters classroom for the first time. The mobile apps requests and get responses from the Main Server, who initiates the notifications to the mobile apps.

If the students does not reach School after a predefined time $t$ after he has boarded the School Bus. The Main Server will initiate a notification or an alert to the Parent's mobile app and this App will allow the Parent to access the location of his ward. Mobile App also allows Parent to access their ward's location at any time whenever required. In case of any Emergency, the mobile App will alert the Parents as well as SPOC, so that they can easily reach help.

\section{SYSTEM ARCHITECTURE AND ENABLING TECHNOLOGY}

The Architecture (Refer Figure 3) consists of various components such as Sensors e.g. RFID, CCTV Camera etc., Getaway Devices, Servers and Mobile Devices and Communication Protocols e.g. Bluetooth, Wi-Fi, Zigbee etc. The students and staff of the school will have an Identity Card in which the RFID tags are embedded. These tags therefore can allow us to uniquely identify the student or the staff member and also their location can be detected. The School Bus and the School Classrooms will have a Gateway Devices as well as various sensors such as RFID Readers, CCTV Cameras and Smoke Detectors. The data from the sensors is gathered and processed by the Gateway Devices. The Gateway Devices acts as a middleware for the proposed application of Internet of Things. A Middleware platform for the IoT provides an abstract layer interposed between the IT infrastructure and the applications [11]. The Middleware platform using Semantic Web Technologies solve many challenges such as Interoperability, Scalability, Abstraction, Spontaneous Interaction, Unfixed Infrastructure, Security and Privacy [12]. Semantic Web approaches can provide context awareness to applications and meaningful information to users so they can decide how to compose multiple services and improve security and privacy decisions [13]. The RFID tags and RFID Readers use the ZigBee protocol for the communication, while the communication between the Gateway Devices and the Main Server is done either by Wi-Fi or by Mobile Internet such as GPRS, 2G or 3G technologies. The Main Server gathers

IJECE Vol. 6, No. 6, December 2016 : 3052-3059 
and processes all the information that it acquires from these multiple Gateway Devices. After the processing these information it can send alerts or notifications to the parents or staff members with the help of native mobile app or a web application installed on their mobile phones. The Cloud Server acts a backup server and the data and the $\log$ is replicated periodically into the Cloud. The Cloud Server will help in recovery management when the Main Server fails. Figure 2 is shows the System Model of Proposed System.

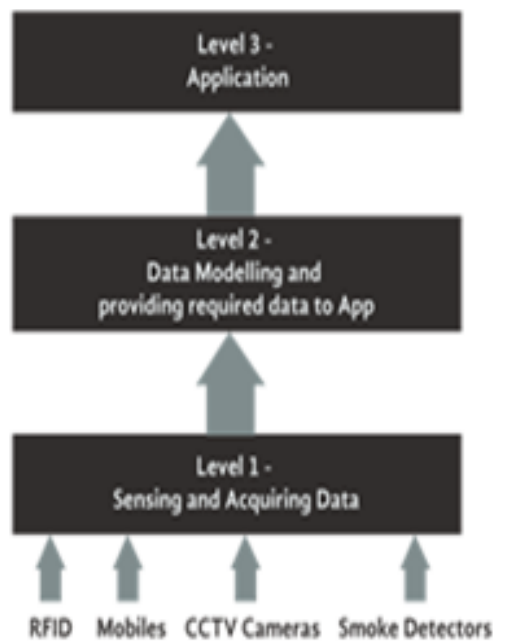

Figure 2. System Model of Proposed System

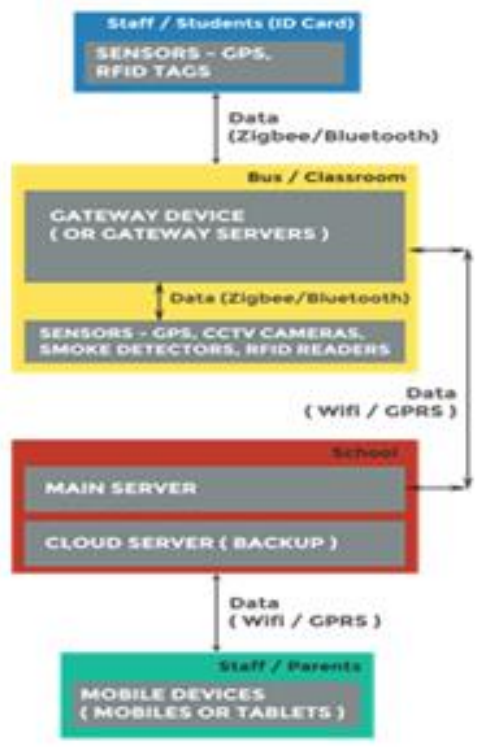

Figure 3. Architecture of Proposed System

\section{WORKING OF THE PROPOSED SYSTEM}

The main functionality of the system is to ensure the safety of the students. Flow of events when a Student boards the School bus. Following is the Algorithm showing the flow of events when a Student boards the School Bus:

a. Set Variable t1(time) when Student Boards the School Bus

b. RFID Reader present in the Bus detects the identity of the Student say s1 and location 11

c. The information processed by the RFID Reader is sent to the Gateway Device present in the Bus

d. The Gateway Devices processes the information and sends the information to the Main Server.

e. The Main Server then processes the information i.e. $t 1, \mathrm{~s} 1$ and 11. It triggers an notification - "Student s1 boarded the School Bus B at $\mathrm{t} 1$ time at 11 location"

f. The Main Server sends the notification to the parent of the student s1 via the Mobile App.

g. The Gateway Device of the Bus checks that "Have all the students S boarded the Bus?" If No, it repeats the Steps 1-6 else, continue to next Step.

h. All the processed information is send to the Main Server

The Main Server logs the information for that Bus.Flow of events when the Student enters the School Classroom. Following is the Algorithm showing the flow of events when a Student enters the School Classroom:

a. Student $\mathrm{s} 1$ enters the School Classroom $\mathrm{c} 1$ at time $\mathrm{t} 2$.

b. RFID Reader present in the Classroom c1 identifies the Student s1.

c. The information processed by the RFID Reader is sent to the nearest Gateway Device.

d. The Gateway Devices processes the information and sends the information to the Main Server.

e. The Main Server then processes the information i.e. t2, s1 and c1. It triggersan notification - "Student s1 entered the School Classroom c1 at t 2 time".

f. The Main Server sends the notification to the parent of the student s1 via the Mobile App.

g. The Gateway Device of the Bus checks that "Have all the students S entered the Classroom C?" If No, it repeats the Steps 1-6 else, continue to next Step.

h. All the processed information is send to the Main Server.

i. The Main Server logs the information for that Classroom. 
Flow of events when the Student does not enter the Classroom within the predefined time period. Following is the Algorithm showing the flow of events when a Student does not enter the Classroom within the predefined time period:

a. Student s1 enters the School Bus.

b. The RFID Reader present in the School Bus identifies the Student.

c. The information processed by the RFID Reader is sent to the Gateway Device. The Gateway Device marks $\mathrm{t} 1$ for this event.

d. The Gateway Devices g1 processes the information received and sendsthe information to the Main Server M.

e. For Every Student s1, the Main Server M checks: if $\mathrm{t} 3=\mathrm{t} 2-\mathrm{t} 1>$ predefined time $\mathrm{t}$, where $\mathrm{t} 2$ is the current time. If Yes, go to Step 8 else go to Step 6.

f. The Main Server checks if Student s1 has entered Classroom c1. If Yes, go to Step 7else go to Step 5.

g. The Main Server logs the information for that Student s1, Bus b1 and Classroom c1.

h. The Main Server will request the location of the School Bus b1 the student had boarded to the Gateway Device $\mathrm{g} 1$ of the Bus b1.

i. Is the School Bus b1 still travelling towards School? If Yes, go to Step 5 else go to Step 10.

j. Activate the emergency mechanism for the concerned Student s1 and Bus b1.

\section{IMPLEMENTATION DETAILS AND RESULTS}

Most of the IoT applications are mobile applications. There are multiple protocols such as HTTP, MQTT, CoAP which can be used for IoT application development. MQ Telemetry Transport Protocol (MQTT) has a numerous advantages over HTTP. MQTT is a lightweight publish/subscribe messaging protocol (used by Facebook Messenger) while HTTP is designed as a request-response protocol for clientserver computing, not necessarily optimized for mobile and push capabilities, particularly in terms of battery usage.

In the mobile environment, response times, throughput, lower battery use and lower bandwidth are key design criteria. If MQTT is compared with HTTP, MQTT gives faster response and throughput, and consumes less battery and bandwidth usage is also less. So it makes MQTT well suited for following criterias such as if connectivity is intermittent, if bandwidth is at a premium, when an enterprise application needs to interact with one or more phone apps, and phone or tablet apps need to send data reliably without requiring code retry logic. Another advantage of MQTT over HTTP is that it is integrated with enterprise messaging middleware, so it works with enterprise-level applications that push data to mobile apps. Using MQTT developers can create mobile applications using HTML and Javascript and yet have the messaging function working at the native layer, in native Java code, deployed on android. Basically, MQTT is designed for low latency, assured messaging and efficient distribution. HTTP is not optimized for low power usage or minimizing the amount of bytes flowing.

MQTT uses TCP is lightweight, and has features beneficial to IoT devices. It is mature and there are a lot of client and server implementations, making it easier to develop upon. MQTT works on a pub/sub architecture. A client subscribes to a channel on a server, and when a server receives new information for that channel, it pushes it out to that device. Another great feature of MQTT is that you can set a priority or Quality of Service (QoS):

a. 0 : The client/server will deliver the message once, with no confirmation required.

b. 1: The client/server will deliver the message at least once, confirmation required.

c. 2: The client/server will deliver the message exactly once by using a handshake process.

\subsection{Comparitive Analysis}

\subsubsection{Packet Size}

The size of data packets in HTTP is up to $2 \mathrm{~Kb}$ is more than the MQTT which is having packet size up to 2bytes. We have captured the MQTT and HTTP data packets using Wireshark tool, to analyse the packet size difference between MQTT and HTTP.

Using MQTT protocol:

We have connected MQTT client to Eclipse server where MQTT broker is installed (MQTT lens). We have connected MQTT client to Eclipse server where MQTT broker is installed (MQTT lens). Figure 4 shos the MQTT client connection. 


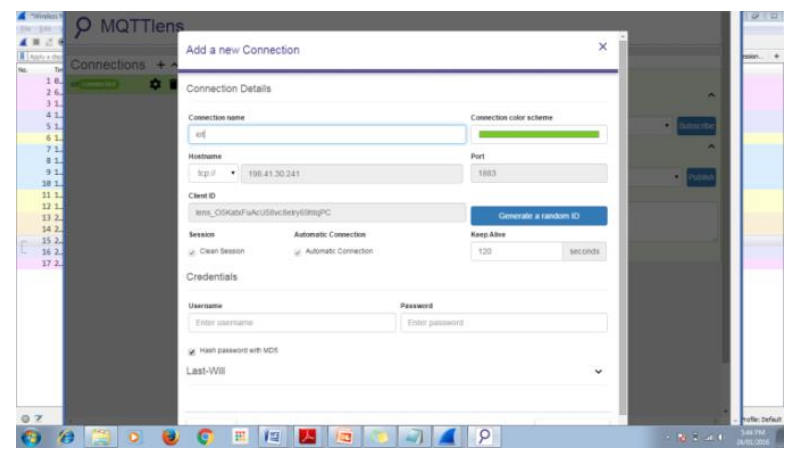

Figure 4. MQTT Client Connection

Then we have captured the MQTT packet using wire shark. Figure 5 clearly shows the data packets sent through the MQTT protocol.

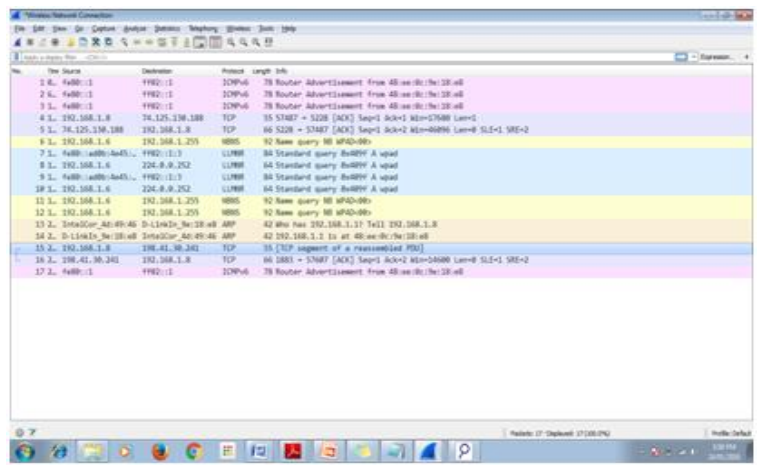

Figure 5. Packet Size

Using HTTP Protocol:

Figure 6 clearly shows the packet size through the HTTP protocol. From the above two packet size figures, its clear that HTTP packet size is more than the MQTT packet size.

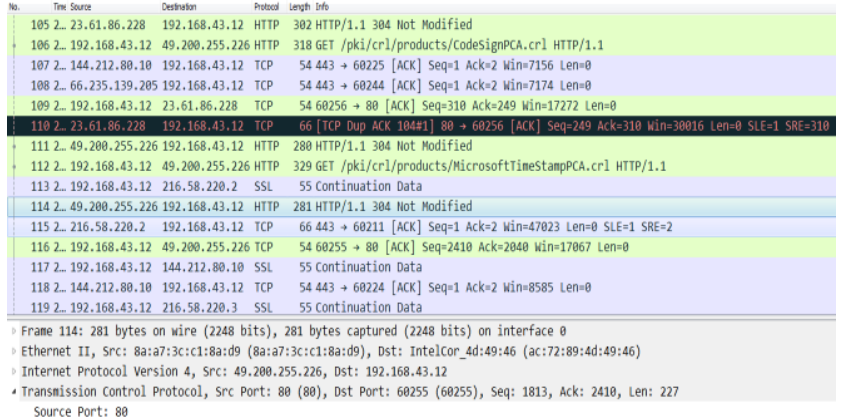

Source Port: 89

Figure 6. Packet Size

\subsubsection{Power Consumption}

While using Http, battery used for creating and maintaining a connection is more than the MQTT protocol. Figure 7 shows the graphical representation of power consumption while using WIFI network. 


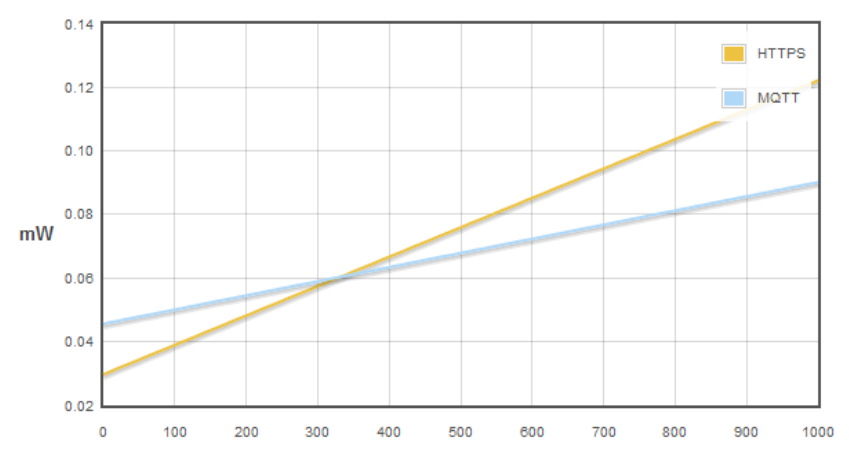

Figure 7. Power Consumption

\subsubsection{Time Latency}

Figure 8 shows the time latency between Http and MQTT protocol. The time delay in fetching or receiving the data is slightly more in Http than MQTT. It is due to the reason of large packet size of Http takes time delay to fetch or receive the data.

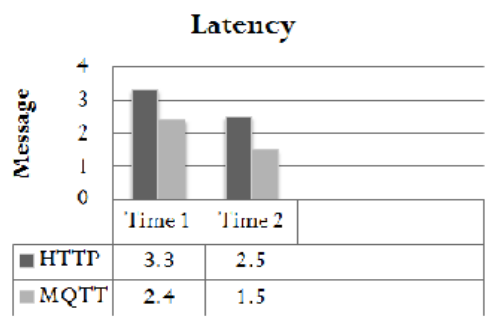

Figure 8. Time Latency

MQTT uses less power to maintain an open connection, to receive messages and to send them. Other advantages built into the MQTT protocol are retained messages and multiple subscriptions 'multiplexed' over one connection. If battery consumption is a key design criteria, then MQTT is the clear answer.

\section{CONCLUSION}

Now a days, researchers are thinking of many applications where electronics devices such as computers, laptops, sensors, mobiles are expected to get connected without intervention of human beings and IoT makes it happen. So in this paper we have used IoT to propose solution for parents concerns about their school going kids so that they will be aware about the location of their kids at any point of time. Parents will also get to know about any emergency situation. To implement this system CCTV camera, mobiles and different sensors such as RFID, smoke detectors need to be used and as per the requirement they will be connected with GPRS, Wi-Fi, Zigbee, Bluetooth. The Details about all devices and communication technologies to be used, is also explained. A system model and architecture of proposed system is also given in this paper. All data collected by different sensors and devices is collected, aggregated and processed to know more about the context to satisfy the purpose correctly and effectively. Further this application can be implemented with the help of MQTT protocol.

\section{REFERENCES}

[1] K. Ashton, "That 'Internet of Things' Thing," http://www.rfidjournal.com/articles/view?4986.

[2] L. Atzori, et al., "The Internet of Things: A survey," in Elsevier, Computer Networks, 2010.

[3] T. Lu and W. Neng, "Future internet: The internet of things," in 3rd International Conference on Advanced Computer Theory and Engineering (ICACTE), vol. 5, 2010.

[4] P. Guillemin and P. Friess, "Internet of things strategic research roadmap," The Cluster of European Research Projects, Tech. Rep., 2009.

[5] http://timstonor.wordpress.com/2013/06/06/smart-cities-why-what-how-how/ 
[6] F. Amato, et al., "The Talking Museum Project", in The 4th International Conference on Emerging Ubiquitous Systems and PervasiveNetworks (EUSPN-2013), 2013.

[7] C. N. Verdouw, et al., "Virtualisation of floricultural supply chains: A review from an Internetof Things perspective" in Elsevier, Computers and Electronics in Agriculture, 2013.

[8] Y. Xu, et al., "The Research of Safety Monitoring System Applied inSchool Bus Based on the Internet of Things," in Elsevier, Advanced in Control Engineeringand Information Science, 2011.

[9] <http://www.zdnet.com/blog/igeneration/uniform-computer-chips-track-student-locations/15681/>

[10] <http://www.pcworld.com/article/2011352/texas-school-uses-rfid-badges-to-track-student-locations.html>

[11] M. A. Chaqfeh and N. Mohamed, "Challenges in Middleware Solutions for the Internet of Things," in International Conference onCollaboration Technologies and Systems (CTS), 2012.

[12] V. Avchat and P. Gupta, "A study of Semantic Middleware for Internet of Things," Internationl Journal of Advanced Research in Computer Science and Software Engineering, vol/issue: 4(12), pp. 1-6, 2014.

[13] Z. Song, et al., "Semantic middleware for the Internet of Things," in Proc. IOT, 2010.

\section{BIOGRAPHIES OF AUTHORS}
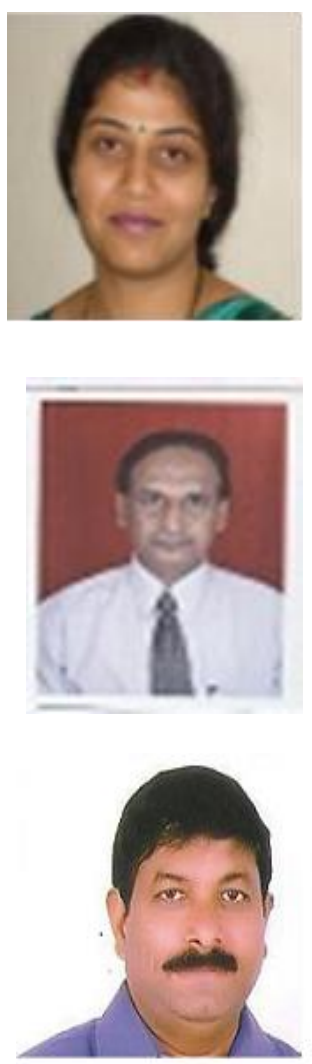

Mrs.Poonam Gupta has completed Bachelor of Engineering in Computer Science and Engineering from Amaravati University and Master of Technology in Computer Engineering from Punjab Technical University in 1998 and 2009 respectively. She is currently working as an Assistant Professor in computer Engineering Department, G. H. Raisoni College of Engineering \& Management, Savitribai Phule Pune University and pursuing Ph. D. I Computer Science and Engineering from K. L. Univeristy, Vijayawada. Her research interests include wireless sensor networks, Internet of Things.

Dr. D D Shah has completed Bachelor of Engineering in Electronics from Banglore University and Master of Engineering in Electronics from Shivaji University, Kolhapur in 1984 and 1992 respectively. He has received his Ph. D. from Indian Institute of Technology, Bombay in 2003. $\mathrm{He}$ is working as Professor in Electronics and Telecommunication Engineering Department, G. H. Raisoni College of Engineering \& Management, Savitribai Phule Pune University. His research interests include Fiber Optic Communication, Image Processing, Wireless Sensor Networks

Dr K V V Satyanarayana has completed Bachelor degree from Andhra University, Visakhapatnam in 1984. His masters in computer Aplication from Andhra University, Visakhapatnam in 1998. And He received his $\mathrm{Ph} \mathrm{D}$ (CSE) in 2011 from Acharya Nagarjuna University, Guntur. His research area includes cloud computing, Biometrics. 